\title{
Renovascular and growth effects of childhood sarcoid
}

\author{
K P Morris, M G Coulthard, P J Smith, A W Craft
}

\begin{abstract}
A boy developed early onset sarcoidosis, an extremely rare mimic of juvenile chronic arthritis. Renal granulomas caused severe hypertension and renal impairment, which was controlled by azathioprine and steroids. Severe uveitis caused visual impairment. Impaired growth and delayed puberty, not previously described in childhood sarcoidosis, required growth hormone and testosterone treatment.
\end{abstract}

(Arch Dis Child 1996;75:74-75)

Keywords: sarcoidosis, hypertension, uveitis, growth failure.

Sarcoid presenting under 4 years is extremely rare, mimicking juvenile chronic arthritis with a rash, uveitis and arthritis, but without pulmonary involvement. ${ }^{1}$ Renal granulomas and granulomatous vasculitis are rare in childhood sarcoidosis, and growth failure has not been described. We describe such a case.

\section{Case history}

An afebrile white boy, aged 2.6 years, presented with painless swelling over the wrists and ankles, effusions in both knees, and an extensive rash resembling eczema. Rheumatoid factor was negative, but his erythrocyte sedimentation rate (ESR) was raised at 75 $\mathrm{mm} /$ hour. He then developed pericarditis which responded to prednisolone, $60 \mathrm{mg} / \mathrm{m}^{2}$ daily. Juvenile chronic arthritis was diagnosed, and maintenance prednisolone continued at 15 $\mathrm{mg} / \mathrm{m}^{2}$ on alternate days. He developed anterior uveitis at 4 years, and sarcoidosis was considered. A wrist synovectomy showed noncaseating granulomas (figure), and Kveim was injected, but not biopsied because there was no visible reaction. Plasma angiotensin converting enzyme activity was normal.

At 8 years he developed severe hypertension that was difficult to control. His glomerular filtration rate (GFR), estimated from plasma creatinine and height, was normal at $105 \mathrm{ml} / \mathrm{min} /$ $1.73 \mathrm{~m}^{2}$. Urine analysis for blood, protein, and excess homovanillic acid have remained negative. A dimercaptosuccinic acid scan was normal, but renal arteriography demonstrated irregular, stenosed peripheral arteries. By 12 years his GFR had fallen to 40 , and he had developed glycosuria and a mild urine concentrating defect.

A renal biopsy specimen showed focal mixed inflammatory infiltrate and fibrosis, tubular atrophy, and non-caseating granulomas, con- firming sarcoidosis (figure). A small artery had mural thickening, and was distorted by a granuloma.

Azathioprine, $60 \mathrm{mg} / \mathrm{m}^{2}$ day, was added to the maintenance prednisolone, and the ESR fell to $13 \mathrm{~mm} /$ hour. His GFR improved to 55 , where it has remained for five years, and he continues to require hypotensive medication. His serum ionised calcium and urine calcium excretion have been normal throughout. At 17 years a reduction in his azathioprine dose caused a rise in ESR which rapidly settled when it was restored.

Despite considerable synovial hypertrophy, there has been little joint destruction and his mobility is only moderately reduced. Despite laser treatment and capsulotomies for severe uveitis, his vision is $6 / 60$ right, 6/18 left. His chest remains normal, with no reduction in carbon monoxide transfer factor.

Between 3 and 10 years his height fell from the 75th centile for age to below the third. Growth hormone treatment increased his growth velocity by about $4 \mathrm{~cm} /$ year, without acceleration in bone age. At 14.4 years he had no signs of sexual development, and he was treated with testosterone, $100 \mathrm{mg} / \mathrm{month}$. By 17 years he had developed secondary sexual characteristics and experienced erections and
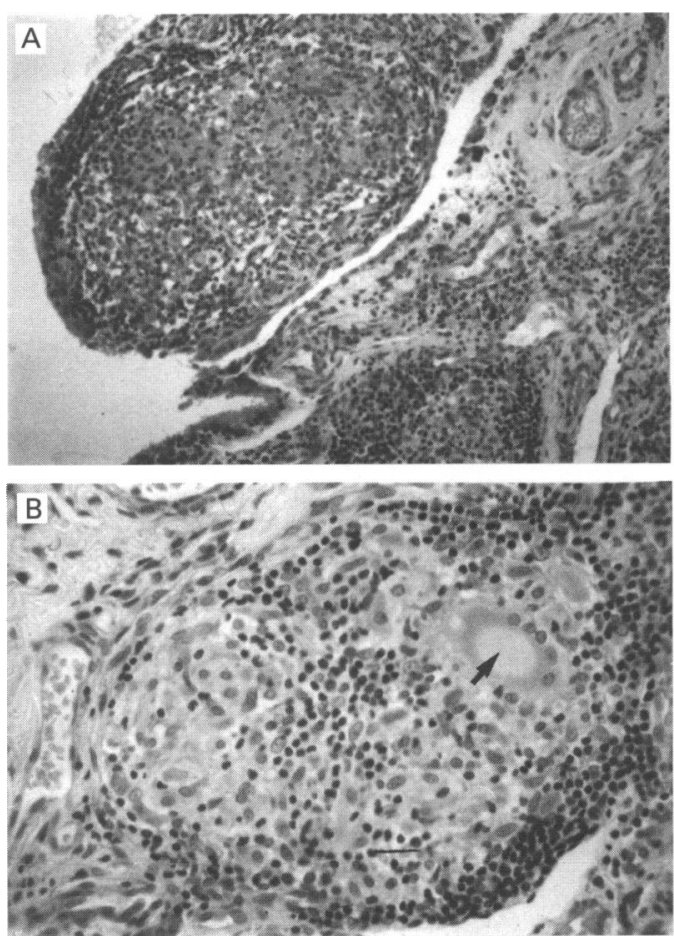

Figure 1 Non-caseating granulomas seen in synovium resected from wrist $(A)$ and in renal biopsy $(B)$; the arrow indicates a multinucleated giant cell. 
emissions, but his serum gonadotrophin concentrations remained prepubertal, and testicular volumes at $3 \mathrm{ml}$. At 18 years his testes are $8 \mathrm{ml}$, and pubertal development has continued without further treatment.

\section{Discussion}

This is the first report of early onset sarcoidosis associated with granulomatous kidney involvement and a vasculopathy. Early onset sarcoidosis is extremely rare; most major textbooks of paediatric rheumatology and nephrology describe only the adult pattern. Our patient illustrates the similarity to juvenile chronic arthritis, though the joint swelling is painless, and the rash differs. ${ }^{1}$ A Kveim test is diagnostic, but must be biopsied, even in the absence of a visible reaction. Plasma angiotensin converting enzyme activity is unhelpful; it may remain normal despite active multisystem disease.

Renal failure in adult sarcoidosis is usually due to hypercalcaemia, hypercalciuria and nephrocalcinosis; none of these occurred in our case. Though renal granulomas occur in up to $27 \%$ of adults with sarcoidosis, ${ }^{2}$ few cause dysfunction, and only two cases have been reported in children. ${ }^{34}$ Several reports suggest that steroid treatment may improve renal function, and that alternate day steroid maintenance is necessary to prevent relapse. ${ }^{3}$ Our case demonstrates that maintenance steroids may not prevent a decline in renal function, but that azathioprine, not previously reported to be used in this condition, arrested the deterioration, and subsequently maintained stable function. Successful treatment may not alter the hypertension because healing can result in further fibrosis.

Neither growth nor puberty failure have been described previously in early onset sarcoidosis, but both are seen in other chronic childhood conditions with unremitting disease activity. His low dose steroid treatment is unlikely to have contributed greatly. Growth hormone treatment appears to have produced benefit to his height, and testosterone allowed him to develop secondary sexual characteristics at an appropriate age.

1 James DG, Kendig EL. Childhood sarcoidosis. Sarcoidosis 1988; 5: 57-9.

2 Romer FK. Renal manifestations and abnormal calcium metabolism in sarcoidosis. $Q \mathcal{F}$ Med 1980; 95: 233-47.

3 Turner MC, Shin ML, Ruley EJ. Renal failure as a presenting sign of diffuse sarcoidosis in an adolescent girl. $A m \mathcal{F}$ Dis Child 1977; 131: 997-1000.

4 Martini A, Serenella Scotta M, Magrini U. Granulomatous sarcoidosis of the kidneys with renal insufficiency in a 12 year old girl. Nephrologie 1980; 1: 117-9. 